PROCEEDINGS OF THE

AMERICAN MATHEMATICAL SOCIETY

Volume 137, Number 1, January 2009, Pages 303-305

S 0002-9939(08)09527-0

Article electronically published on July 8, 2008

\title{
A NOTE ON ALMOST DISJOINT FAMILIES
}

\author{
PÉTER KOMJÁTH
}

(Communicated by Julia Knight)

\begin{abstract}
We give a short proof of the existence of arbitrarily large chromatic almost disjoint systems. With the same method we solve a problem of P. Szeptycki.
\end{abstract}

One of the questions posed by P. Erdős and A. Hajnal in 2 was, do there exist arbitrarily large chromatic almost disjoint systems of countable sets? This was eventually proved by G. Elekes and G. Hoffmann in 11. Later the result was extended to systems of sets of larger cardinality ([3]). These proofs applied a Baire category type argument in a topological setting.

Here we give a short, direct proof of the Elekes-Hoffmann-Komjáth theorem. With the same method we settle a problem of P. Szeptycki (4]). He asked if it is consistent that for every family $\mathcal{H}$ of almost disjoint countable subsets of $\mathbb{R}$ there are sets $B_{0}, B_{1}, \ldots \subseteq \mathbb{R}$ such that every $H \in \mathcal{H}$ has $1 \leq\left|H \cap B_{i}\right|<\omega$ for some $i<\omega$.

Notation and definitions. We apply the standard axiomatic set theory notation. If $V$ is a set and $\mu$ is a cardinal, then $[V]^{\mu}=\{X \subseteq V:|X|=\mu\}$. If $X, Y$ are sets, then ${ }^{X} Y$ denotes the set of functions from $X$ to $Y$. A set system $\mathcal{H} \subseteq[S]^{\mu}$ is almost disjoint if $|A \cap B|<\mu$ holds whenever $A, B \in \mathcal{H}, A \neq B$. The chromatic number $\mathrm{Chr}(\mathcal{H})$ of some set system $\mathcal{H}$ on the underlying set $S$ is the least cardinality $\kappa$ such that there is a coloring $f: S \rightarrow \kappa$ so that no $H \in \mathcal{H}$ gets monocolored.

Theorem 1 ([1, [3]). If $\kappa \geq \mu$ are infinite cardinals, then there exists an almost disjoint system $\mathcal{H} \subseteq[S]^{\mu}$ for some $|S|=2^{\kappa}$ with $\mathrm{Chr}(\mathcal{H})>\kappa$.

Proof. Set $\Phi=\left\{f: \alpha \rightarrow \kappa, \alpha<\kappa^{+}\right\}$. Clearly $|\Phi|=2^{\kappa}$. For some of the functions $f \in \Phi$ we define $H(f) \in[\Phi]^{\mu}$ as follows. Let $f: \alpha \rightarrow \kappa$. If it is possible to find a set $X \subseteq \alpha$ of order type $\mu$, cofinal in $\alpha$ (and so $\operatorname{cf}(\alpha)=\operatorname{cf}(\mu)$ ) such that $f$ is constant on $X$, then set $f \in \Phi^{*}$ and let $H(f)=\{f \mid \beta: \beta \in X\}$ for one such $X$. If no such set $X$ can be found, make $f \notin \Phi^{*}$ and leave $H(f)$ undefined. Our system is $\mathcal{H}=\left\{H(f): f \in \Phi^{*}\right\}$.

Lemma 1. $\mathcal{H}$ is almost disjoint.

Proof. Assume that $|H(f) \cap H(g)|=\mu$. Then $H(f)=\{f \mid \gamma: \gamma \in X\}$ and $H(g)=$ $\{g \mid \delta: \delta \in Y\}$ where $f: \alpha \rightarrow \kappa, g: \beta \rightarrow \kappa, X \subseteq \alpha, Y \subseteq \beta$ are cofinal subsets of

Received by the editors November 20, 2007.

2000 Mathematics Subject Classification. Primary 03E05.

Key words and phrases. Almost disjoint sets.

This research was partially supported by Hungarian National Research Grant T 046991.

(C)2008 American Mathematical Society Reverts to public domain 28 years from publication 
ordinal $\mu$. By our assumption, for a cofinal subset $X^{\prime} \subseteq X$ it is true that if $\gamma \in X^{\prime}$, then there is a $\delta \in Y$ such that $f|\gamma=g| \delta$. This implies that $f \mid \gamma \subseteq g$ and as $X^{\prime}$ is cofinal in $\alpha, f \subseteq g$. Reversing the argument we get that $g \subseteq f$, so $f=g$.

Lemma 2. $\operatorname{Chr}(\mathcal{H})>\kappa$.

Proof. Let $F: \Phi \rightarrow \kappa$ be a coloring. By transfinite recursion on $\alpha<\kappa^{+}$we define the functions $\left\{f_{\alpha}<\kappa^{+}\right\}$such that $f_{\alpha}: \alpha \rightarrow \kappa$ and $f_{\beta} \subseteq f_{\alpha}$ for $\beta<\alpha$. Set $f_{0}=\emptyset$ and let $f_{\alpha}=\bigcup\left\{f_{\beta}: \beta<\alpha\right\}$ if $\alpha$ is a limit. If $f_{\alpha}$ is already determined, then let $f_{\alpha+1}$ be that extension of $f_{\alpha}$ which has $f_{\alpha+1}(\alpha)=F\left(f_{\alpha}\right)$. Clearly, there is a unique function $f: \kappa^{+} \rightarrow \kappa$ such that $f_{\alpha}=f \mid \alpha$ for every $\alpha<\kappa^{+}$. As $\kappa^{+}$is regular, $f^{-1}(i)$ is cofinal for some $i<\kappa$. Let $X$ be the set of the first $\mu$ elements of $f^{-1}(i)$, $\alpha=\sup (X)$. $X$ witnesses that the required condition holds for $f_{\alpha}$. Therefore $f_{\alpha} \in \Phi^{*}$ and a set $H\left(f_{\alpha}\right) \subseteq\left\{f_{\beta}: \beta<\alpha\right\}$ is chosen using some $X^{\prime}$ which may be different from $X: H\left(f_{\alpha}\right)=\left\{f_{\beta}: \beta \in X^{\prime}\right\}$. But then, $f_{\alpha}(\beta)=j$ for all $\beta \in X^{\prime}$ and so $F\left(f_{\beta}\right)=f(\beta)=j\left(\beta \in X^{\prime}\right)$. Therefore, $H\left(f_{\alpha}\right)$ is monocolored by $F$.

The lemmas conclude the proof of the theorem.

Theorem 2. There is an almost disjoint family $\mathcal{H} \subseteq[\mathbb{R}]^{\omega}$ such that there are no sets $B_{i} \subseteq \mathbb{R}(i<\omega)$ such that for every $H \in \mathcal{H}, 1 \leq\left|H \cap B_{i}\right|<\omega$ holds for some $i<\omega$.

Proof. It suffices to construct a family on any set of cardinality the continuum. Set $\Phi=\left\{f: \alpha \rightarrow{ }^{\omega} 2, \alpha<\omega_{1}\right\}$. Clearly $|\Phi|=2^{\aleph_{0}}$ and our system will be a family of subsets of $\Phi$.

Assume that $f: \alpha \rightarrow \omega_{2}$ is given, where $\alpha<\omega_{1}$ is a limit. Partition $\omega=$ $X(f) \cup Y(f)$ as follows. For $i<\omega$, add $i$ to $X(f)$ if $\gamma_{i}=\sup \{\beta<\alpha: f(\beta)(i)=1\}$ is less than $\alpha$; otherwise add $i$ to $Y(f)$. We do not define $H(f)$ unless $\gamma=\sup \left\{\gamma_{i}\right.$ : $i \in X(f)\}<\alpha$. In this case let $\alpha_{0}<\alpha_{1}<\cdots$ be a sequence converging to $\alpha$ such that $\gamma<\alpha_{0}$ and for every $i \in Y(f)$ the set $\left\{\alpha_{n}: f\left(\alpha_{n}\right)(i)=1\right\}$ is infinite. This is possible by the definition of $Y(f)$. Set $H(f)=\left\{f \mid \alpha_{n}: n<\omega\right\}$ and let $\Phi^{*}$ be the set of those elements of $\Phi$ for which the above condition holds. Then $\mathcal{H}=\left\{H(f): f \in \Phi^{*}\right\}$ is a system of countable subsets of $\Phi$.

Lemma 3. $\mathcal{H}$ is almost disjoint.

Proof. Assume that $f \neq g \in \Phi^{*}$ and $|H(f) \cap H(g)|=\omega$. Then $H(f)=\left\{f \mid \alpha_{n}: n<\right.$ $\omega\}$ and $H(g)=\left\{g \mid \beta_{n}: n<\omega\right\}$ where $\alpha_{n} \rightarrow \alpha, \beta_{n} \rightarrow \beta$ and $\alpha, \beta$ are the domains of $f, g$, respectively. If $f\left|\alpha_{n}=g\right| \beta_{m}$, then necessarily $\alpha_{n}=\beta_{m}$ and $f\left|\alpha_{n}=g\right| \beta_{m}$. If this holds for infinitely many $n$ and $m$, then clearly $\alpha=\beta$ and $f=g$.

Assuming now that $B_{i} \subseteq \Phi$ for $i<\omega$, we are going to find an $H(f) \in \mathcal{H}$ such that for no $i$ does $1 \leq\left|H(f) \cap B_{i}\right|<\omega$ hold.

Construct the sequence $\left\{f_{\alpha}: \alpha<\omega_{1}\right\}$ as follows: $f_{0}=\emptyset$. If $\alpha$ is a limit, let $f_{\alpha}=\bigcup\left\{f_{\beta}: \beta<\alpha\right\}$. Finally, if $f_{\alpha}$ is given, let $x_{\alpha} \in{ }^{\omega} 2$ be the following function: $x_{\alpha}(i)=1$ if $f_{\alpha} \in B_{i}$; otherwise $x_{\alpha}(i)=0$ and let $f_{\alpha+1}$ be that unique function on $\alpha+1$ with $f_{\alpha+1} \supseteq f_{\alpha}$ and $f_{\alpha+1}(\alpha)=x_{\alpha}$.

For $i<\omega$ define $A_{i}=\left\{\beta: f_{\beta} \in B_{i}\right\}$. Now $A_{0}, A_{1}, \ldots$ are various subsets of $\omega_{1}$. Put $i \in X$ if $A_{i}$ is countable and then set $\gamma_{i}=\sup \left(A_{i}\right)<\omega_{1}$. If $A_{i}$ is uncountable, let $i \in Y$. Set $\gamma=\sup \left\{\gamma_{i}: i \in X\right\}$. Let $\alpha<\omega_{1}$ be a limit ordinal with $\gamma<\alpha$ such that every $A_{i}$ with $i \in Y$ is cofinal in $\alpha$. There are ordinals that satisfy this; actually those ordinals form a closed, unbounded subset of $\omega_{1}$. 
Now observe that $X\left(f_{\alpha}\right)=X, Y\left(f_{\alpha}\right)=Y$, and the condition on the boundedness of $\gamma$ also holds, so $f_{\alpha} \in \Phi^{*}$; moreover $H\left(f_{\alpha}\right) \cap B_{i}=\emptyset$ for $i \in X$ and $\left|H\left(f_{\alpha}\right) \cap B_{i}\right|=\omega$ for $i \in Y$.

\section{REFERENCES}

1. G. Elekes, G. Hoffmann: On the chromatic number of almost disjoint families of countable sets, Infinite and finite sets (Colloq., Keszthely, 1973), Colloq., Math. Soc. János Bolyai, 10, North-Holland, Amsterdam, 1975, 397-402. MR0373905 (51:10105)

2. P. Erdős, A. Hajnal: On a property of families of sets, Acta Math. Acad. Sci. Hungar., 12(1961), 87-123. MR0150047 (27:50)

3. P. Komjáth: Dense systems of almost-disjoint sets, Finite and infinite sets (Eger, 1981), Colloq. Math. Soc. János Bolyai, 37, North-Holland, Amsterdam, 1984, 527-536. MR818255 (87f:04005)

4. Paul J. Szeptycki: Transversals for strongly almost disjoint families, Proc. Amer. Math. Soc., 135(2007), 2273-2282. MR2299505

Department of Computer Science, Eötvös University, P. O. Box 120, Budapest, 1518, HUNGARY

E-mail address: kope@cs.elte.hu 